Journal of Computer Science 7 (10): 1554-1559, 2011

ISSN 1549-3636

(C) 2011 Science Publications

\title{
Consensus Decision Making on Scale-Free Buyer Networks
}

\author{
${ }^{1}$ Sunantha Sodsee ${ }^{1}$ Maytiyanin Komkhao and ${ }^{2}$ Phayung Meesad \\ ${ }^{1}$ Department of Mathematics and Computer Science, \\ Fernuniversitaet in Hagen, Germany \\ ${ }^{2}$ Department of Information Technology, \\ King Mongkut's University of Technology, \\ North Bangkok, Thailand
}

\begin{abstract}
Problem statement: Due to a behavior of buyers effects to a growth of marketing, then a group of agreement of buyers and buyers' behavior on eventually placing orders are investigating. Approach: In this study, a discrete-time consensus protocol for a scale-free buyer networks is proposed. Herein, an initial purchase intention of each buyer is defined to be active or non-active and an impulse signal is used to activate a purchase intention as an advertisement. Results: To investigate a global decision making in the buyer networks, the number of active buyers after activating was considered. Finally, the numerical simulations were conducted to visualize the effectiveness of the proposed protocol. The results showed that buyers change their intentions based on activation of advertisement that also effect to the group decision making of network. Conclusion: The active buyers still be active if the current number of active buyers $(\varphi(\mathrm{x}))$ is more than or equal to the defined value of their $\mathrm{th}_{\mathrm{i}}$. On the other hand, the non-active buyers still be non-active if the current number of non-active buyers $n-\varphi(x))$ is more than or equal to the defined value of their the $h_{i}$ Otherwise, their states are altered.
\end{abstract}

Key words: Decision making, consensus protocol, scale-free networks, advertisement, numerical simulations, buyer networks, swarm intelligence, heterogeneous structure, world networks, rendezvous problems

\section{INTRODUCTION}

Nowadays, the group (coordination or swarm) behavior of multi-agent systems and their exhibited swarm intelligence have attracted a great research interest of researchers from fields of engineering and biology, such as birds flocking, robot swarming, fish schooling, to name just a few. Consensus problem is related to group coordination, which is for a group of agents to make a decision or to reach an agreement, depending on their shared state information, where information exchange among the agents, or say, the topology of the networked multi-agent system, plays a key role for consensus. Based on the topology, the goal is to design a suitable protocol for a group to reach a consensus. A consensus protocol is a communication rule for exchanging the state information between an agent and its neighbors so as to reach consensus, which is a kind of a distributed decision making, because each agent has just local information of its neighbors (Bauso et al., 2009). The consensus protocol has also been applied to solve many problems in group (distributed) decision making such as the synchronization of coupled oscillators, flocking and swarming, formation control and rendezvous problems.

Here, the consensus protocol results in a distributed decision making. It has an advantage over centralized decision making in the sense that a decision maker is not required to access information from all other decision makers. Its result in group agreement also seems to a brainstorming among group members (Lin, 2009; Lin, 2010). Due to the effectiveness of the distributed decision making, it has been applied to deal with the problems of inventory control, which is important to study an activation effecting to purchasing decision making for instance, Guneri et al. (2009) studied the influence of children on family purchasing decision making. To deal with the consensus protocol, Bauso and his colleagues have been proposed distributed consensus protocols to coordinate orders of a group of buyers. They studied how the information exchange among buyers and the coordination of the buyers impact final orders. In addition, they also presented a protocol for cooperative inventory control of retailers, which leads to the best coordination between retailers for ordering products.

Corresponding Author: Sunantha Sodsee, Department of Mathematics and Computer Science, Fernuniversitaet in Hagen, Germany 
In this study, a discrete-time consensus protocol is proposed for a group of buyers to reach an agreement based on scale-free buyer networks. The buyer networks have scale-free features because of the expanded size of buyer networks. They can be increased continuously according to a power-law distribution of connectivity. The state of buyer represents the buyer's purchase intention. It is updated according to the states and degrees of itself and its neighbors. Buyers have different degrees, which measure their popularities and the size of networks is expanded based on the preferential attachment. To investigate buyers' behaviors on eventually placing orders, both active and non-active buyers are considered. An impulse signal is used as an advertisement in order to activate a purchase intention of buyers.

The rest of this study is organized as follows. The next part gives concepts of scale-free networks and consensus problem on graphs. The discrete consensus protocol is proposed in buyer networks, later. Numerical simulations are carried out to show benefits of proposed work and finally, a conclusion and discussion are provided.

\section{MATERIALS AND METHODS}

Herein, we first introduce some basic concepts of scale-free networks, notation of algebraic graph theory and consensus protocol. Later, our proposed work in buyer networks will be presented in details.

Scale-free networks: Most real systems in nature can be represented as complex networks, where nodes are the elements of a system and links represent the interactions in between. Examples are numerous, such as an organization is a network of people, the World Wide Web is a network of websites and a market is a network of interactions between procedures and consumers.

In 1950s, Erdös and Rényi described a network with complex topology by a random graph. They introduced a random network model. Starting with $n$ nodes, each pair of nodes is connected with a probability generating a random network. Due to many very large-scale complex networks are not completely random. In 1998, Watts and Strogatz (WS) introduced a concept of small-world network. The WS model begins with a one-dimensional lattice of $n$ nodes with links between the nearest and the next-nearest neighbors. Each link is rewired with a probability in order to shift one end of link to a new node chosen at random. Focusing on the connectivity distribution of both ER and WS networks, it peaks at an average value and decay exponentially. Then, they are called exponential networks or homogenous networks, because each node has the same number of link connections.
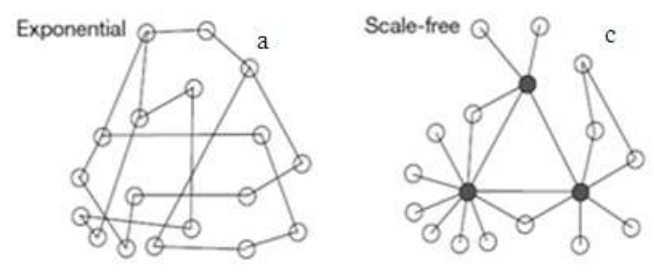

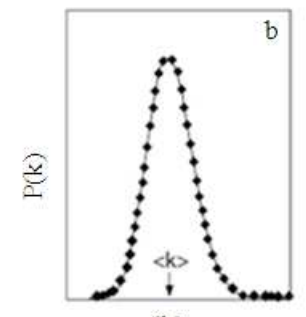

(k)

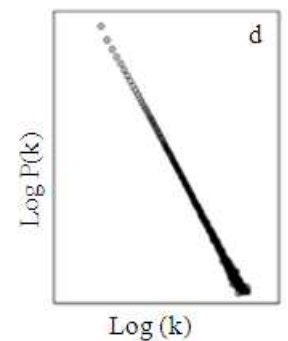

$\log (\mathrm{k})$
Fig. 1: Connectivity distributions (Jeong et al., 2000)

The connectivity distribution can be viewed as a measure of nodes popularity. The real large-scale complex networks have the nature that the connectivity probability distribution $\mathrm{P}(\mathrm{k})$ of a node connecting to $k$ other nodes is a power-law degree distribution, $\mathrm{P}(\mathrm{k})$ $\mathrm{k}^{-\gamma}$, where $\mathrm{k}$ is the degree of a node and $\gamma$ is a scalar exponent. Many large-scale complex networks are scale-free. Thus, the existing network models such as ER model and WS model fail to incorporate the following two major characters of real complex networks. Firstly, the networks are continuously growing by the addition of new nodes and secondly, the new nodes connect preferentially to highly existing connected nodes.

The network connectivity of ER networks is depicted in Fig. 1c. It is characterized by $P(k)$, denoting that a node has $\mathrm{k}$ links. $\mathrm{P}(\mathrm{k})$ peaks at $\mathrm{k}=\langle\mathrm{k}\rangle$ and degrees distribute exponentially for large $\mathrm{k}$. It is shown in Fig. 1d that most nodes in the scale-free networks have a few links/degrees, but few nodes have a very large number of degrees, hence for a large $\mathrm{k}, \mathrm{P}$ (k) decays as a power-law.

The scale-free networks has the heterogeneous structure reflecting the nature of most real world networks, in case that networks are open, the number of nodes can be increased continuously. In addition, the probability of a new node connecting to the existing nodes is not constant, but dependent on the degrees of the nodes. To construct networks with the scale-free properties, they can be built in the following way. Starting with a small number of nodes $\left(\mathrm{m}_{0}\right)$ at each time step, a new node with $m$ links $\left(m \leq m_{0}\right)$ is added to the existing graph and connected to the existing nodes (j) in terms of the preferential attachment with assuming that 
$\mathrm{m}_{0}=\mathrm{j}$ at the starting time. The preferential attachment of node $\mathrm{i}$ is given by its connectivity $\left(\mathrm{k}_{\mathrm{i}}\right)$ :

$\prod_{\mathrm{i}}=\frac{\mathrm{k}_{\mathrm{i}}}{\sum_{\mathrm{j}} \mathrm{k}_{\mathrm{j}}}$

where, $k_{i}$ is the degree of node $i$, the sum runs over $j$ existing nodes in the system.

In this study, the purchase behavior of a group of buyers is concerned. Buyer networks have the scalefree properties (Tseng et al., 2008). Due to the social characteristics, some buyers have larger connectivity and each buyer can connect with each other based on its preference. In addition, the network size is expanded as to the power-law degree distribution.

Consensus on Graph: Here, the interaction of a complex network of agents is represented by an undirected graph. Denote $A_{n}=\left[a_{i j}\right] \in R^{n \times n}$ as the adjacency matrix of graph $G_{n}=\left(V_{n}, E_{n}\right)$, where $V_{n}$ is the set of agents $v_{i}, I=\{1,2, \ldots, n\}$ and $E_{n}$ the set of edges $\rho_{\mathrm{ij}}=\left(\mathrm{v}_{\mathrm{i}}, \mathrm{v}_{\mathrm{j}}\right), \mathrm{i}, \mathrm{j}=\{1,2, \ldots, \mathrm{n}\}$ and:

$$
a_{i j}=\left\{\begin{array}{lc}
1, & \text { if } v_{i}, v_{j} \in E_{n}, \\
0, & \text { Otherwise. }
\end{array}\right.
$$

The neighborhood of agent $\mathrm{i} \mathrm{N}_{\mathrm{i}}$, is defined as $N_{i}=\left\{v_{j} \mid a_{i j} \neq 0\right.$ and $\left.i \neq j\right\}$. The degree matrix of $G$ is $a$ diagonal matrix $D_{n}=\left[d_{i j}\right]$. Where $d_{i j}=0$ for all $i \neq j$ and $\mathrm{d}_{\mathrm{ii}}=\operatorname{deg}\left(\mathrm{v}_{\mathrm{i}}\right)$ is the degree of $\mathrm{v}_{\mathrm{i}}$. Further, the graph Palladian $\mathrm{L}$ associating with the undirected graph $\mathrm{G}$ is defined as $\mathrm{L}=\mathrm{D}-\mathrm{A}$, where:

$$
l_{i j}= \begin{cases}d_{i i}, & \text { if } i=j, \\ -1, & \text { if } i \neq j \text { and } v_{j} \text { is connected to } v_{i} \\ 0, & \text { Otherwise. }\end{cases}
$$

The dynamics of agent $\mathrm{i}$ is described by:

$$
\mathrm{x}_{\mathrm{i}}(\mathrm{t}+1)=\mathrm{x}_{\mathrm{i}}(\mathrm{t})+\mathrm{u}_{\mathrm{i}}(\mathrm{t})
$$

where, $x_{i} \in R^{n}$ denotes the state of the agent $i$ at time $t$ and $u_{i} \in R^{n}$ is the control input at time $t$ and $u_{i l}(t)$ is given by:

$\mathrm{u}_{\mathrm{i}}(\mathrm{t})=\varepsilon \sum_{\mathrm{j} \in \mathrm{N}_{\mathrm{i}}} \mathrm{a}_{\mathrm{ij}}\left(\mathrm{x}_{\mathrm{j}}(\mathrm{t})-\mathrm{x}_{\mathrm{i}}(\mathrm{t})\right)$

Substituting (3) into (2) yields: $\mathrm{x}_{\mathrm{i}}(\mathrm{t}+1)=\left(\mathrm{x}_{\mathrm{i}}(\mathrm{t})+\varepsilon \sum_{\mathrm{j} \in \mathrm{N}_{\mathrm{i}}} \mathrm{a}_{\mathrm{ij}}\left(\mathrm{x}_{\mathrm{j}}(\mathrm{t})-\mathrm{x}_{\mathrm{i}}(\mathrm{t})\right)\right.$

Where:

$0<\varepsilon \leq \frac{1}{\Delta}=$ A parameter, in which

$\Delta=$ The maximum degree of nodes, $\Delta=\max _{\mathrm{i}} \sum_{\mathrm{i} \neq \mathrm{j}} \mathrm{a}_{\mathrm{ij}}$

Further, (4) can be recast as:

$x(t+1)=P x(t)$

Where, $P=I-\varepsilon L$ is the Perron matrix of graph $G$, which is a non-negative matrix and $\mathrm{I}$ is the identity matrix.

A matrix is irreducible if its associated graph is strongly connected. A matrix is called row (or column) stochastic if all of its row-sums (or column-sums) are 1. An irreducible stochastic matrix is primitive if it has only one eigenvalue with maximum modulus.

Let $\mathrm{P}$ be a primitive-nonnegative matrix and denote $\mathrm{W}$ and $v$ as left and right eigenvectors, respectively. Then $\mathrm{Pv}=\mathrm{v}, \mathrm{w}^{\mathrm{T}} \mathrm{P}=\mathrm{w}^{\mathrm{T}}$ and $\mathrm{v}^{\mathrm{T}} \mathrm{w}=1$, thus $\lim _{t \rightarrow \infty} \mathrm{P}^{\mathrm{t}}=\mathrm{vw}^{\mathrm{T}}$.

A consensus in discrete-time is asymptotically reached for all initial states if the $\lim _{t \rightarrow \infty} \mathrm{P}^{t}$ exists. This limit exists when $P$ is a primitive matrix. Thus (5) can be recast as $\lim t \rightarrow \infty \mathrm{x}(\mathrm{t})=\mathrm{vw}^{\mathrm{T}} \mathrm{x}(0)$, when $\mathrm{P}=\mathrm{vw}^{\mathrm{T}}$. When $\mathrm{t} \rightarrow \infty$ and $\mathrm{v}=1$, then $(5)$ can be recast as $\mathrm{x}_{\mathrm{i}}=\mathrm{w}^{\mathrm{T}}$ $x(0)$ for all $i$. A group of agents is said to reach a global consensus if $x_{j}(t)=x_{j}(t)$ for each pair $(i, j), i, j=1,2, \ldots$, $\mathrm{n}$ and $\mathrm{i} \neq \mathrm{j}$. The common agreement value of all agents is called the group decision value, which is $x_{\mathrm{i}} \rightarrow \mathrm{a}$. Thus $\lim t \rightarrow \infty \mathrm{a}(\mathrm{t})=\sum_{\mathrm{i}} \mathrm{w}_{\mathrm{i}} \mathrm{x}_{\mathrm{i}}(0)$ with $\sum_{\mathrm{i}} \mathrm{w}_{\mathrm{i}}=1$.

For an undirected graph, one has $\mathrm{a}_{\mathrm{ij}}=\mathrm{a}_{\mathrm{ji}}$ and $\mathrm{l}_{\mathrm{ij}}=\mathrm{l}_{\mathrm{ji}}$ for all $i \neq j$. Hence the undirected graph $G$ is balanced or $\mathrm{p}$ is doubly-stochastic, then one has $\mathrm{w}_{\mathrm{i}}=\frac{1}{\mathrm{n}}$ and thus:

$\lim _{t \rightarrow \infty} \mathrm{a}(\mathrm{t}) \mathrm{w}_{\mathrm{i}}=\frac{\sum_{\mathrm{i}} \mathrm{x}_{\mathrm{i}}(0)}{\mathrm{n}}$

Therefore, $\mathrm{P}$ is a column stochastic matrix with a left eigenvector of $\mathrm{w}_{\mathrm{i}}=\frac{1}{\mathrm{n}} 1$, then the group decision as $\mathrm{t} \rightarrow \infty$ is given as:

$\mathrm{a}=\frac{1}{\mathrm{n}} 1^{\mathrm{T}} \mathrm{x}(0)$ 
Buyer networks: Buyer networks consist of buyers and the information exchange relationship between them. The state of buyer $i$ or $x_{i}(i=1,2 \ldots, n)$ represents the purchase intention: active or non-active. The buyers can exchange their purchase intention information with each other if and only if they are linked.

The buyer networks are scale-free. They expand continuously and a new buyer connects other existing ones based on the preferential attachment. The interaction of buyers is undirected.

Consensus problems have been investigated based on various network topologies, such as random networks, small-world networks and scale-free networks (Wang and Guo, 2008). Wang and Guo studied a discrete-time consensus model on the scale-free networks, where the affect of network topology to the consensus is considered. The state of each agent is updated according to the degrees and states of its neighbors and the neighborhood is regarded as a probabilistic domain. The neighborhood is defined in terms of the probability, i.e., $j$ is the neighbor of $i$ only if $i$ and $j$ are adjacent to each other with a probability, however, $\mathrm{i}$ and $\mathrm{j}$ do not definitely communicate to each other. This neighborhood concept is different from the graph theory in that if $j$ is the neighbor of $i$, there is a link between $i$ and $j$ and $i$ and $\mathrm{j}$ can communicate with each other.

The state of buyer $\mathrm{i}$ is updated by degrees and the states of its neighbors at instant $t$ in the following way:

$$
x_{i}(t+1)=\frac{x_{i}(t) k_{i}+\sum_{j \in N_{i}} x_{j}(t) k_{j}}{k_{i}+\sum_{j \in N_{i}} k_{j}}
$$

Where, $x_{i}(t)$ is the state of buyer $i$ at instant $t, k_{i}$ is its degree/popularity and $\mathrm{N}_{\mathrm{i}}$ is the set of neighbors of buyer i. It is remarked that the purchase intention of each buyer is depended on the intention of its neighbors and its neighbors' popularity.

To consider the purchase behavior of buyers, the local information exchange is concerned. The information of buyer $i$ consists of an initial state/purchase intention $\left(\mathrm{x}_{\mathrm{i}}(0)\right)$, threshold value $\left(\mathrm{th}_{\mathrm{i}}\right)$ to be defined in the following and popularity/degree of connection $\left(\mathrm{k}_{\mathrm{i}}\right) \cdot \mathrm{x}_{\mathrm{i}}(0)$ is randomly chosen to be 0 or 1 with $\mathrm{x}_{\mathrm{i}}(0)=0$ implying a non-active buyer and $\mathrm{x}_{\mathrm{i}}(0)=1$ an active one. The non-active buyer is the one who does not want to buy anything, while the active buyer is the one who wants to buy. Further, $\mathrm{th}_{\mathrm{i}}$ is an integer taken randomly between 1 and the size of network. The th presents the number of active buyers with whom each buyer can accept to coordinate with.

The number of active buyers can be defined as:

$\lim _{t \rightarrow \infty} \varphi(x)=\operatorname{nAve}(x(0))$
Where, Ave $(x(0))$ is the average value of the initial purchase intention of buyers. Thus, the number of nonactive buyers is $n-\varphi(x)$.

To activate the purchase intention of buyers, the unit impulse signal $\delta\left(\mathrm{t}-\mathrm{t}_{\mathrm{f}}\right)$ is simulated as the advertisement, $\delta\left(t-t_{\mathrm{f}}\right)=\left\{\begin{array}{c}1,\left(\mathrm{t}-\mathrm{t}_{\mathrm{f}}\right) \\ 0, \text { otherwise }\end{array}\right.$ where $\delta\left(\mathrm{t}-\mathrm{t}_{\mathrm{f}}\right)=1$ means that there is an advertisement, which activates the purchase intention of buyers.

At the activation time $t_{f}$, if the current number of active buyers $\varphi(x)$ is less than the threshold value $t_{i}$ of any activated active buyers, then their present states will be changed. In contrast, the activated non-active buyers will change their states when $n-\varphi(x)<t_{i}$.

\section{RESULTS}

Here, the group decision making of buyers will be simulated. At the activation time $\left(\mathrm{t}_{\mathrm{f}}\right)$, the current $\varphi(\mathrm{x})$ is compared to the $\mathrm{th}_{\mathrm{i}}$ of activated buyers in order to change their states.

Figure 2 depicts a scale-free buyer network, in which $\mathrm{m}_{0}$ is $3, \mathrm{~m}$ is 2 and the average degree is 2 . The network consists of 6 buyers and 6 links. Each buyer has different degree or popularity. The information of each buyer is listed in Table 1 .

Supposing $\varepsilon=\frac{1}{4}$ for the consensus among buyers at time $t$, the graph Laplacian L and Perron matrix P are:

$$
\mathrm{L}=\left|\begin{array}{cccccc}
1 & -1 & 0 & 0 & 0 & 0 \\
-1 & 3 & -1 & 0 & -1 & 0 \\
0 & -1 & 2 & 0 & -1 & 0 \\
0 & 0 & 0 & 1 & -1 & 0 \\
0 & -1 & -1 & -1 & 4 & -1 \\
0 & 0 & 0 & 0 & -1 & 1
\end{array}\right|
$$

And:

$$
\mathrm{P}=\mathrm{I}-\frac{1}{4} \mathrm{~L}
$$

For the buyer network shown in Fig. 2, it has different initial purchase intention, i.e., there are two non-active buyers and four active ones.

The simulation result is depicted in Fig. 3. It is shown that the group agreement is reached around at $t=$ 20 and the group decision value is approximately 0.67 , which means that the group agreement of this buyer network for purchasing is at a probability of $67 \%$.

Table 1: Information of buyers

\begin{tabular}{lllllll}
\hline Buyers (i) & 1 & 2 & 3 & 4 & 5 & 6 \\
\hline $\mathrm{x}_{\mathrm{i}}(0)$ & 0 & 1 & 0 & 1 & 1 & 1 \\
$\mathrm{th}_{\mathrm{i}}$ & 6 & 1 & 6 & 2 & 5 & 2 \\
$\mathrm{k}_{\mathrm{i}}$ & 1 & 3 & 2 & 1 & 4 & 1 \\
\hline
\end{tabular}


Due to the balanced buyer network, the left eigenvector of $\mathrm{P}$ is $\mathrm{w}=\frac{1}{\mathrm{n}} 1$ where 1 is the identity matrix and $n=6$, then:

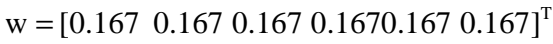

The group decision value is derived as:

$$
\begin{aligned}
& \lim t \rightarrow \infty \\
& +0.167 \mathrm{x}_{3}(0)+0.167 \mathrm{x}_{4}(0) \\
& \left.+0.167 \mathrm{x}_{5}(0)+0.167 \mathrm{x}_{6}(0)\right]=0.668
\end{aligned}
$$

Or:

$$
\lim _{\mathrm{t} \rightarrow \infty} \mathrm{a}(\mathrm{t})=\frac{\sum_{\mathrm{i}} \mathrm{x}_{\mathrm{i}}(0)}{6}=0.668
$$

It is remarked that this is consistent with the simulation results.

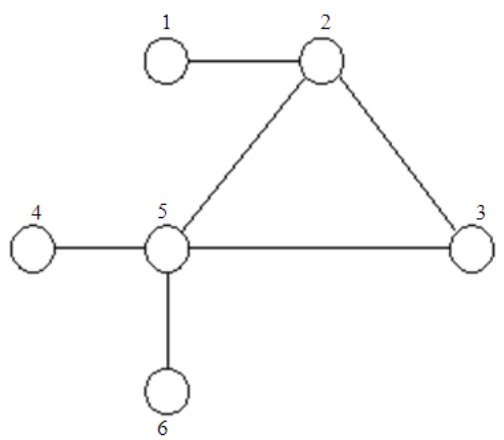

Fig. 2: Scale-free buyer network

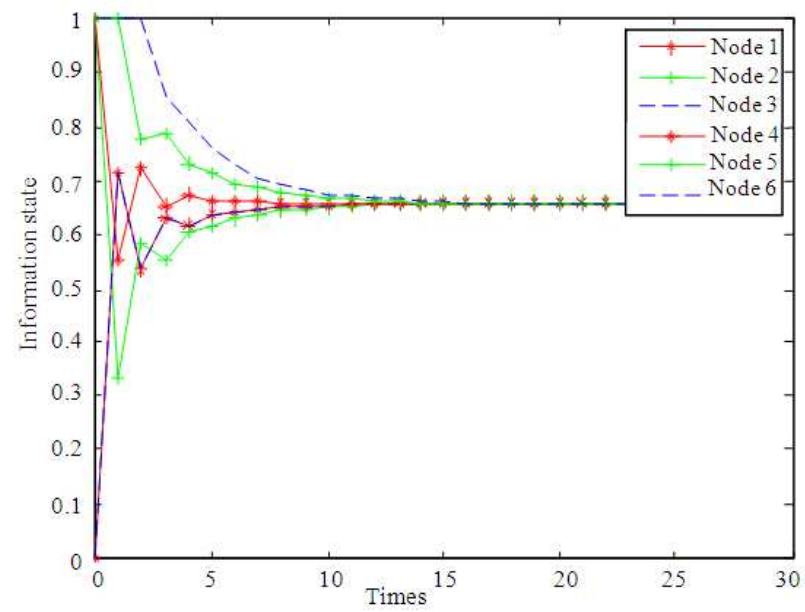

Fig. 3: Group decision making among buyers

\section{DISCUSSION}

To investigate the buyers' behavior, the active buyers are first activated at time $t_{f}=10$. The simulation results are shown in Fig. 4a presenting $\varphi(x)$ and Fig. 4b depicting a $(\mathrm{t})$. It is shown that, as a consequence of activation, buyer 5 changes its intention. Its state is changed from active to non-active due to $\varphi(x)<t_{5}$. In this case, the advertisement influences the buying behavior of buyer 5 . Then the number of active buyers in the network $(\varphi(x))$ is consequently changed from four to three (Fig. 4a) and the final group consensus $(\mathrm{a}(\mathrm{t}))$ is also changed from around 0.67 to about 0.5 (Fig. 4b).

Similarly, activating the non-active buyers at time $t_{\mathrm{f}}=10$. It is shown in Fig. 5 that buyers 1 and 3 want to buy the products due to $n-\varphi(x)<t_{i}$. Then, their purchase intentions are changed from non-active to active. The number of active buyers in the network $(\varphi(x))$ is consequently changed from four to six (Fig. 5a) and the final consensus $(\mathrm{a}(\mathrm{t}))$ is also changed from around 0.67-1 (Fig. 5b). It means that the group agreement is reached for all.

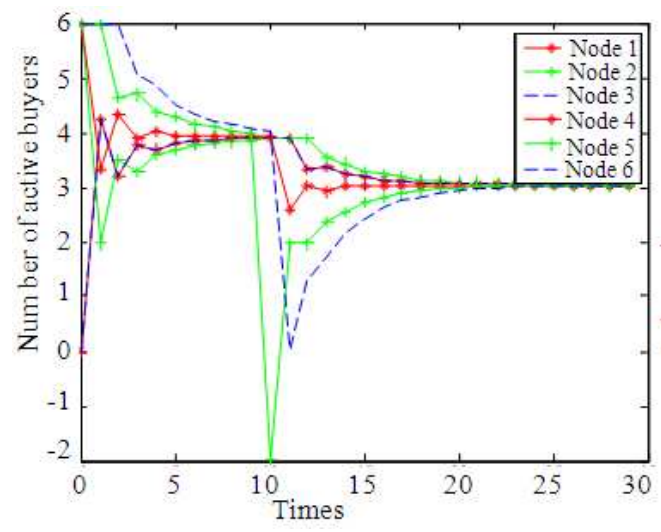

(a)

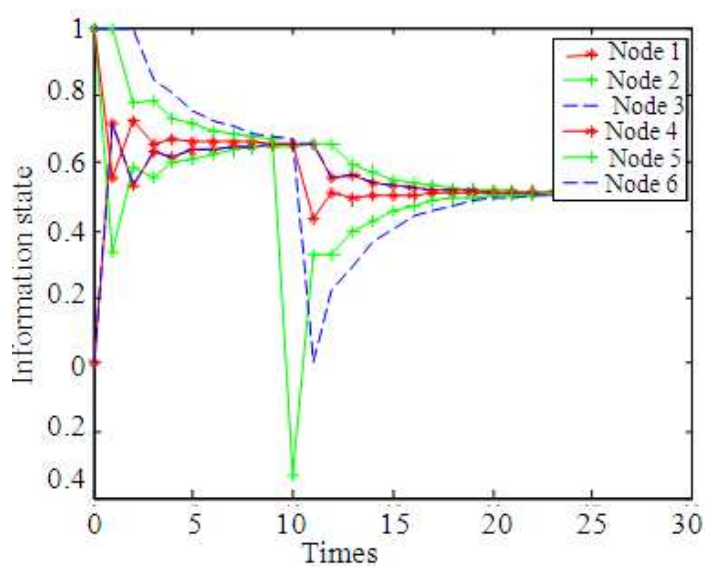

(b)

Fig. 4: Group decision making with activated active buyers 


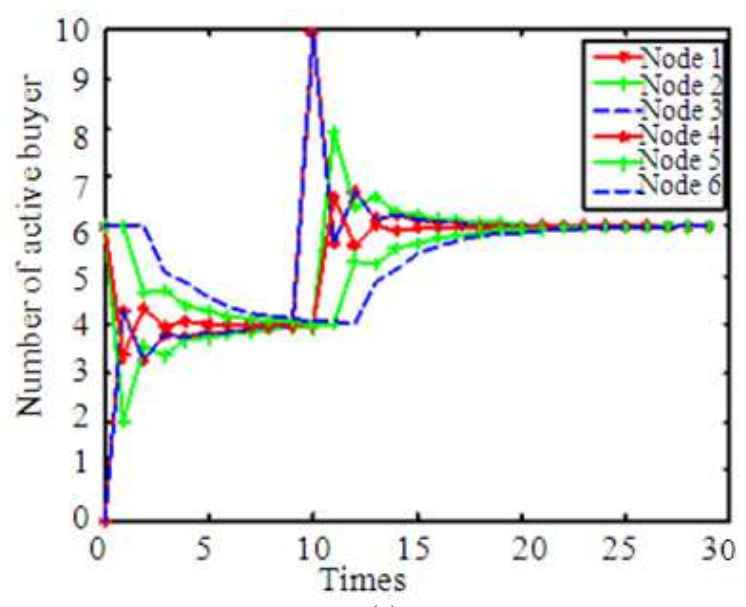

(a)

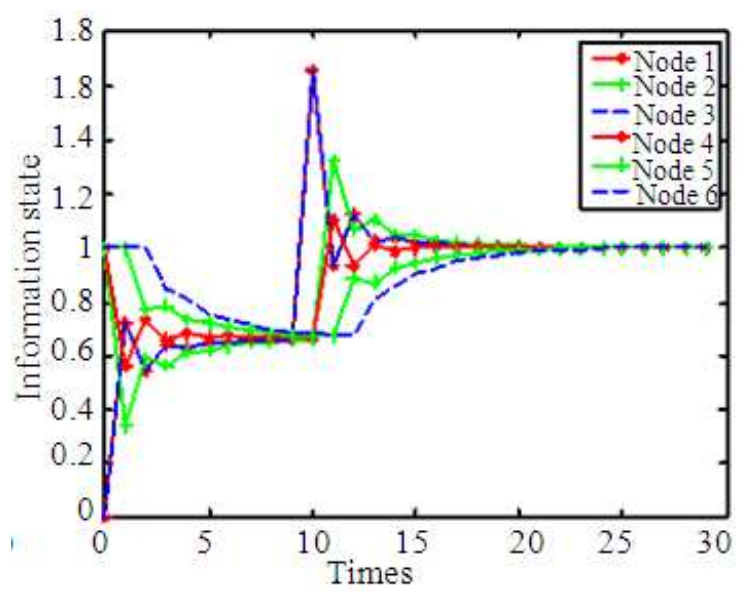

(b)

Fig. 5: Group decision making with activated nonactive buyers

\section{CONCLUSION}

In this study, a discrete-time consensus protocol for scale-free buyer networks is concerned in order to study a purchase intention of buyers. Herein, an initial purchase intention of each buyer is defined to be active or non-active. An impulse signal is acting as the advertisement to activate a purchase intention. To show its effectiveness, simulations for the group decision making among buyers and the purchase intention activation in active buyers and non-active ones have been conducted. The active buyers still be active if the current number of active buyers $(\varphi(x))$ is more than or equal to the defined value of their $t_{\mathrm{i}}$. On the other hand, the non-active buyers still be non-active if the current number of non-active buyers $n-\varphi(x))$ is more than or equal to the defined value of their $t_{\mathrm{i}}$. Otherwise, their states are altered. When the purchase intention of each buyer is changed, it affects to the group decision making of network.

\section{REFERENCES}

Bauso, D., L. Giarr'em and R. Pesenti, 2009. Distributed consensus in noncooperative inventory games. Eur. J. Operat. Res., 192: 866-878. DOI: 10.1016/J.EJOR.2007.10.012

Guneri, B., O. Yurt, M.D. Kaplan and M. Delen, 2009. The influence of children on family purchasing decisions in Turkey. Asian J. Market., 2: 20-32. http://scialert.net/abstract/?doi=ajm.2009.20.32

Jeong, H., B. Tombor, R. Albert, Z.N. Oltvai and A. Barabasi, 2000. The large-scale organization of metabolic networks. Nature, 407: 651-654. DOI: $10.1038 / 35036627$

Lin, H.T., 2009. A multifunctional system for supporting collaborating works and decision making. Inform. Technol. J., 8: 49-56. http://docsdrive.com/pdfs/ansinet/itj/2009/4956.pdf

Lin, J., 2010. Information systems for enhancing customer relationships. Inform. Technol., J., 9: 1306-1316.

http://docsdrive.com/pdfs/ansinet/itj/2010/13061316.pdf

Tseng, J., S. Chen, S. Wang and S. Li, 2008. Scale-Free Networks Emerged in the Markets: Human Traders Versus Zero-Intelligence Traders. In: Agent-Based Approaches in Economic and Social Complex Systems, Terano, T., H. Kita and S. Takahashi (Eds.). Springer, Japan, pp: 245-254. ISBN: 443187433X

Wang, H. and Y. Guo, 2008. Consensus on scale-free network. Proceedings of the 2008 American Control Conference, Jun. 11-13, Seattle, WA, pp: 748-752. DOI: 10.1109/ACC.2008.4586582 